\title{
Two New Lignans from the Bark of Zanthoxylum planispinum
}

\author{
Yun Hu, Yu Chen, Yue Shang, and Guangzhong Yang* \\ Laboratory for Natural Product Chemistry, College of Phamacy, South Central Lniversitv for Nationalities, \\ Whan +3007t. P. R. China. "E-mail: vanggz888, $126 . \mathrm{com}$ \\ Received February 15, 2009, Accepted June 24, 2009
}

Key Words: Zanthoxulum planispinum. Lignan. Planispine A and B

Zanthorylum planispintm Sieb. et Zucc. has been widely used as a folk medicine for clearing away cold. preventing toothache and expelling roundworms. ' Chemical constituents of Zanthoxy/hm have been studied extensively. Previous phytochemical investigations on this species revealed that benzophenanthridine alkaloids. ${ }^{2}$ coumarins. ${ }^{3}$ amides $^{+}$and lignians are largely represented in this genus. Chemical studies of the bark of this plant have never been conducted previously. As continuation of our chemical studies of ethnomedicinal plants. ${ }^{6}$ we have investigated the bark of $Z$. planispinmm and reported herein the isolation and structure elucidation of two new furofuran lignans planispine A (1) and B (2). Their structures were established on the basis of various spectroscopic analy'ses including ID- $\left({ }^{1} \mathrm{H},{ }^{13} \mathrm{C}\right.$ NMR and DEPT) and 2D-NMR (HSQC. HMBC and ROESY) techriques and by comparison of their spectral data with those of related compounds.

Compound 1 was isolated as colorless oil. Its molecular formular was established as $\mathrm{C}_{25} \mathrm{H}_{31} \mathrm{O}_{6}\left(m: z+49.1942[\mathrm{M}+\mathrm{Na}]^{-}\right.$, calcd for 449.1940) on the basis of its HR-ESI-MS analyses. The ${ }^{1} \mathrm{H}$ NMR spectrum showed two $\mathrm{CH}$ group at $\hat{o}_{\mathrm{H}} 2.8+(\mathrm{lH}$. in) and $3.39\left(1 \mathrm{H}\right.$. m). two benzylic $\mathrm{OCH}$ moieties at $\hat{\delta}_{\mathrm{H}} 4.38$ $(1 \mathrm{H}, \mathrm{d} J=7.2 \mathrm{~Hz})$ and $4.83(1 \mathrm{H}, \mathrm{d} J=5.7 \mathrm{~Hz}$ ) two oxygenated $\mathrm{CH}_{2}$ group at $\delta_{\mathrm{H}}+11(1 \mathrm{H}, \mathrm{d}, J=9.3 \mathrm{~Hz})$ and $3.82(1 \mathrm{H}, \mathrm{m})$. $3.78(1 \mathrm{H}, \mathrm{m})$ and $3.24(\mathrm{lH} . \mathrm{dd} . J=8.7 .8 .7 \mathrm{~Hz})$. six aromatic

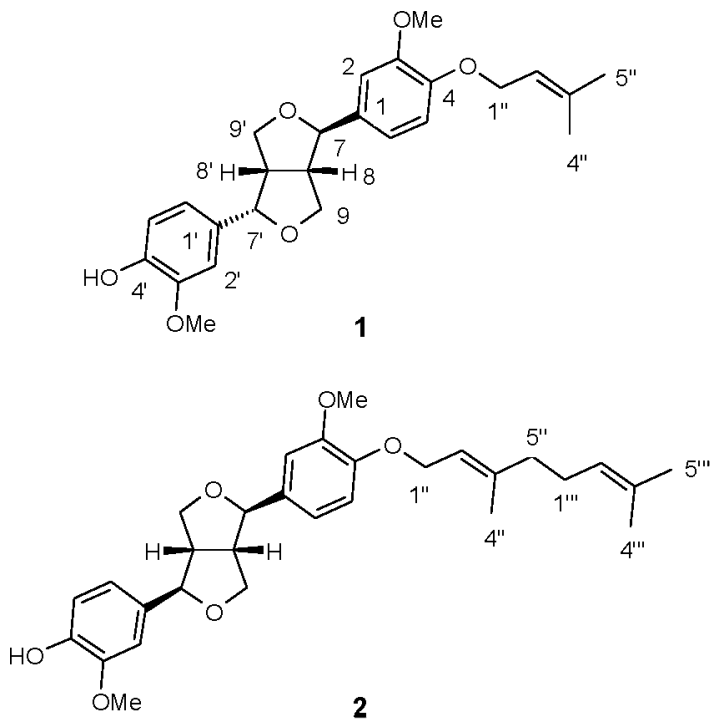

Figure 1. Their structures of compounds 1 and 2 . protons as two $\mathrm{ABX}$ systems at $\hat{\delta}_{\mathrm{H}} 6.79-6.95$ indicating the presence of two 1.2.4-trisubstituted benzene rings. in addition to two methoxy groups at $\delta_{\mathrm{H}} 3.80,3.83$ (each $3 \mathrm{H}, \mathrm{s}$ ). one hydroxy group at $\hat{\delta}_{\mathrm{H}} 7.53(\mathrm{lH}$. s). and one prenyloxy group at $\delta_{\mathrm{H}}+.53(2 \mathrm{H} . \mathrm{d}, J=6.7 \mathrm{~Hz}) .5 .47(1 \mathrm{H} . \mathrm{t}, J=6.7 \mathrm{~Hz}) .1 .72(3 \mathrm{H}$. s). $1.75(3 \mathrm{H}, \mathrm{s})$. Accordingly, compound 1 was assigned to be a lignan of the furofuran type bearing one hydrosy, two methoxy and a prenyloxy group. ${ }^{5, ?}$ This assumption was further supported by the ${ }^{13} \mathrm{C}$ NMR signal including $2 \times \mathrm{CH}(\hat{\mathrm{o}}$ : 55.3 and 50.5$) .2 \times$ benzylic $\mathrm{OCH}(\delta .88 .0$ and 82.3$), 2 \times$ oxygenated $\mathrm{CH}_{2}\left(\hat{\mathrm{d}}_{c}-71.1\right.$ and 69.8$), 6 \times$ aromatic $\mathrm{C}$ and $6 \times$ aromatic $\mathrm{CH}$. The ${ }^{\mathrm{l}} \mathrm{H}$ NMR and ${ }^{13} \mathrm{C}$ NMR data of compound 1 was similar to that of epipinoresinol. ${ }^{8}$ suggesting one hydroxy of epipinoresinol was replaced by a prenyloxy group in $\mathbf{1}$ which was supported by MS fragments at $m z 358\left(\mathrm{M}-\mathrm{C}_{3} \mathrm{H}_{8}\right)$ and $69\left(\mathrm{C}_{5} \mathrm{H}_{9}\right)$. In $\mathrm{HMBC}$ spectrum. the correlations of $\overrightarrow{\mathrm{d}}_{\mathrm{H}} 7.53$

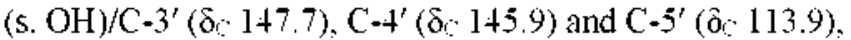
$\hat{\delta}_{\mathrm{H}} 3.80\left(\mathrm{~s} . \mathrm{OCH}_{3}\right) / \mathrm{C}-3\left(\hat{\alpha}_{-}-150.4\right) . \hat{\delta}_{\mathrm{H}} 3.83\left(\mathrm{~s} . \mathrm{OCH}_{3}\right) / \mathrm{C}-3^{\prime}$ and $\delta_{\mathrm{H}} \mathrm{H}_{2}-1^{\prime \prime} / \mathrm{C}-4\left(\delta_{\mathrm{C}} 148.5\right)$ indicated that two methosy. one hydroxy and one prenylosy groups were located at C-3. C-3', $C-t^{\prime}$ and $C-4$ respectively. Furthermore the ROESY spectrum allowed us to confirm the position of the methosy group, showing that both methoxyl signal at $\hat{\partial}_{\mathrm{H}} 3.80$ and 3.83 can correlate with $\mathrm{H}-2$ and $\mathrm{H}-2^{\prime}$ respectively. The relative configuration of 1 was determined on the basis of a ROESY experiment (see Fig. 2). Although compound 1 exhibited the same NMR data as (+)-epipinoresinol ${ }^{8 \mathrm{a}}\left([\alpha]_{\mathrm{D}}=+79\right.$ (c 0.1 , $\mathrm{MeOH})$ ), which has the absolute configuration $7 S^{\prime} 7^{\prime} R, 8 R, 8^{\prime} R$. its optical rotation was opposite. Accordingly. the absolute configuration was assigned as $7 R, 7 S_{1}, 8 S, 8 S$. Thus. the

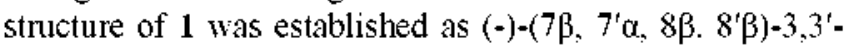
dimethoxy-4-preny loxy -7.9⿱㇒. $: 7^{\prime}, 9$-dieposy lignan-4'-ol with

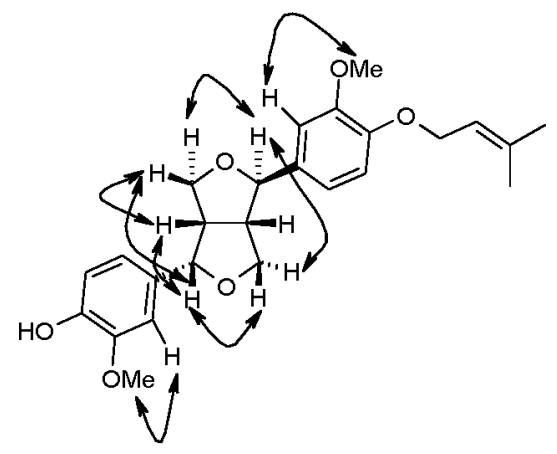

Figure 2. Key NOE correlations for compound 1. 
$(7 R, 7 \mathrm{~S}, 8 \mathrm{~S}, 8 \mathrm{~S})$, named as planispine $\mathrm{A}$.

Compound 2 was obtained as colorless oil. and had a molecular formular $\mathrm{C}_{310} \mathrm{H}_{38} \mathrm{O}_{6}$ from the positive HR-ESI-MS ( $m: 517.2556[\mathrm{M}+\mathrm{Na}]^{+}$. calcd for 517.2566 ). Most of the ${ }^{1} \mathrm{H}$ NMR and ${ }^{13} \mathrm{C}$ NMR signals of 2 were similar to those of $\mathbf{1}$. The miajor spectroscopic differences between them as followed: i) the presence of a geranyloxy group in 2. instead of preny loxy group in the former: $i i)$ more downfield shifted for $\mathrm{C}-7^{\prime}\left(\hat{o}_{c}\right.$ 86.1). C-8 $\left(\delta_{c} 54.9\right) . C-9^{\prime}\left(\delta_{0}, 71.9\right)$ and $\mathrm{C}-1^{\prime}\left(\delta_{C} 135.1\right)$ : iii $)$ Small coupling constants were observed for $\mathrm{H}-7(J=4.5 \mathrm{~Hz})$ and $\mathrm{H}-7^{\prime}(J=4.2 \mathrm{~Hz})$. All over the data suggested the structure of compound 2 was similar to that of pinoresinol. ${ }^{9}$ except one hydroxy of pinoresinol replaced by a geranyloxy group in $\mathbf{2}$ which was supported by MS fragments at $m z 358\left(\mathrm{M}-\mathrm{C}_{10} \mathrm{H}_{16}\right)$ and $137\left(\mathrm{C}_{11} \mathrm{H}_{17}\right)$. The substitued pattern was unambiguously confirmed by $\mathrm{HMBC}$ data. Correlations were oberved between $\hat{o}_{\mathrm{H}} 7.58(\mathrm{~s} . \mathrm{OH}) / \mathrm{C}-3^{\prime}\left(\hat{o}_{c} 147.9\right), \mathrm{C}-4^{\prime}\left(\hat{o}_{c} 1+6.5\right)$ and $\mathrm{C}-5^{\prime}\left(\hat{o}_{c} c\right.$ $115.2), \delta_{\mathrm{H}} 3.81\left(\mathrm{~s} . \mathrm{OCH}_{3}\right) / \mathrm{C}-3\left(\hat{\mathrm{o}}_{\mathrm{C}}, 150.4\right)$. $\hat{\mathrm{o}}_{\mathrm{H}} 3.84\left(\mathrm{~s}, \mathrm{OCH}_{3}\right) /$ $\mathrm{C}-3^{\prime}$ and $\mathrm{H}_{2}-\mathrm{I}^{\prime \prime} / \mathrm{C}-4$ ( $\hat{\delta}_{\mathrm{C}}$ 148.4), confirming two methoxy one hydroxy and one gerany loxy groups were located at C-3. C-3'. $\mathrm{C}-4^{\prime}$ and $\mathrm{C}-4$ respectively. Furthemore. the ROESY spectrum allowed us to confirm the position of the methoxy group. showing that both methoxyl signal at $\hat{\delta}_{\mathrm{H}} 3.81$ and $3.84 \mathrm{can}$ correlate with $\mathrm{H}-2$ and $\mathrm{H}-2^{\prime}$ respectively. The relative configuration of 2 was determined on the basis of a ROESY

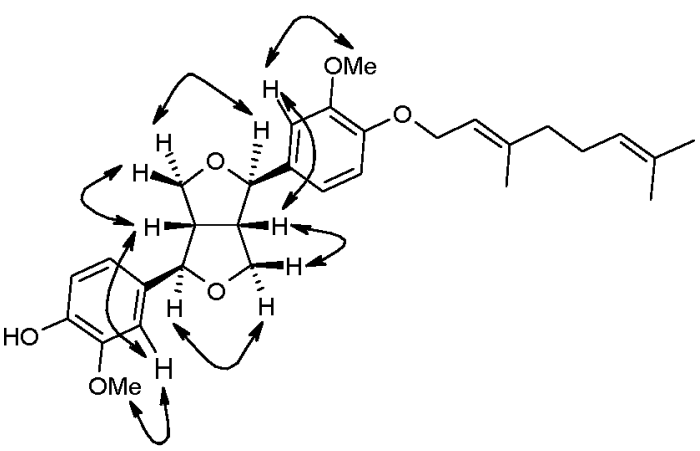

Figure 3. Key NOE correlations for compound 2.

experiment (see Fig. 3). On the basis of levorotatory nature and relative configuration. the absolute configuration of 2 nust be the same as that of (-)-pinoresinol monomethyl ether $^{8 \mathrm{a}}\left([\alpha]_{D}=-58(\mathrm{c} 0.05, \mathrm{EtOH})\right)$. Therefore. the structure of 2 was established as $(-)-\left(7 \beta .7^{\prime} \beta, 8 \beta .8^{\prime} \beta\right)-3.3^{\prime}$-dimethosy-4gerany loxy-7. $9^{\prime}: 7^{\prime} .9$-diepoxylignan- $4^{\prime}$-ol with $\left(7 R, 7^{\prime} R, 8 S\right.$, 8 'S). named as planispine $B$.

\section{Expelimental Section}

Reagent and equipment. Thin-layer cluromatogaphy (TLC): Pre-coated silica gel $G F_{25+}$ plates (Oingdao Haivang Chemical

Table 1. ${ }^{1} \mathrm{H},{ }^{13} \mathrm{C}$ NMR and HMBC data of compound 1 and 2 in acetone

\begin{tabular}{|c|c|c|c|c|c|c|}
\hline \multirow{2}{*}{ position } & \multicolumn{3}{|c|}{1} & \multicolumn{3}{|c|}{2} \\
\hline & ${ }^{\mathrm{l}} \mathrm{H}-\mathrm{NMR}$ & ${ }^{13} \mathrm{C}-\mathrm{NMR}$ & HMBC & ${ }^{\mathrm{j}} \mathrm{H}-\mathrm{NMR}$ & ${ }^{13} \mathrm{C}-\mathrm{NMR}$ & HMBC \\
\hline 1 & & $135.2(s)$ & & & $133.7(\mathrm{~s})$ & \\
\hline 2 & $6.99(\mathrm{~d}, 2.4)$ & $110.5(\mathrm{~d})$ & $C-1,3,4,6,7$ & $6.98(\mathrm{~d}, 1.5)$ & $110.2(d)$ & \\
\hline 3 & & $150.4(\mathrm{~s})$ & & & $150.4(\mathrm{~s})$ & \\
\hline 4 & & $148.5(\mathrm{~s})$ & & & $148.4(\mathrm{~s})$ & \\
\hline 5 & $6.79-6.85(\mathrm{~m})$ & $115.1(\mathrm{~d})$ & & $6.85(\mathrm{~m})$ & $1140(\mathrm{~d})$ & \\
\hline 6 & $6.82-6.92(\mathrm{~m})$ & $118.7(\mathrm{~d})$ & & $6.84(\mathrm{~m})$ & $118.6(d)$ & \\
\hline 7 & $4.38(\mathrm{~d}, 7.2)$ & $88.0(\mathrm{~d})$ & $\mathrm{C}-1,2,6,8,9$ & $4.67(\mathrm{~d}, 4.5)$ & $86.2(\mathrm{~d})$ & $C-2,6,8,9$ \\
\hline 8 & $2.84(\mathrm{~m})$ & 55.3 (d) & $\mathrm{C}-1,7,7^{\prime}, 8^{\prime}, 9^{\prime}$ & $3.09(\mathrm{~m})$ & 54.9 (d) & \\
\hline $9 a$ & $3.82(\mathrm{~m})$ & $71.1(\mathrm{t})$ & & $3.80(\mathrm{~m})$ & $71.8(t)$ & C $-7,8$ \\
\hline $9 \mathrm{~b}$ & $4.11(\mathrm{~d}, 9.3)$ & & & $4.20(\mathrm{~m})$ & & \\
\hline$l^{\prime}$ & & $1309(s)$ & & & $135.1(\mathrm{~s})$ & \\
\hline $2^{\prime}$ & $7.00(\mathrm{~d}, 2.4)$ & 109.7 (d) & $C-1^{\prime}, 3^{\prime}, 4^{\prime}, 6^{\prime}, 7^{\prime}$ & $6.98(\mathrm{~d}, 1.5)$ & $110.7(\mathrm{~d})$ & \\
\hline $3^{\prime}$ & & $147.7(\mathrm{~s})$ & & & $147.9(\mathrm{~s})$ & \\
\hline $4^{\prime}$ & & $145.9(s)$ & & & $146.5(\mathrm{~d})$ & \\
\hline $5^{\prime}$ & $6.90-6.95(\mathrm{~m})$ & $113.9(\mathrm{~d})$ & & $6.79(\mathrm{~m})$ & $115.2(d)$ & \\
\hline $6^{\prime}$ & $6.82-6.92(\mathrm{~m})$ & $118.7(\mathrm{~d})$ & & $6.82(\mathrm{~m})$ & $119.2(\mathrm{~d})$ & \\
\hline $7^{\prime}$ & $4.83(\mathrm{~d}, 5.7)$ & $82.3(\mathrm{~d})$ & $C-1^{\prime}, 2^{\prime}, 6^{\prime}, 8^{\prime}, 9^{\prime}$ & $4.70(\mathrm{~d}, 4.2)$ & $86.1(d)$ & $C-2^{\prime}, 6^{\prime}, 8^{\prime}, 9^{\prime}$ \\
\hline $8^{\prime}$ & $3.39(\mathrm{mi})$ & $50.5(\mathrm{~d})$ & C-9 & $3.09(\mathrm{mi})$ & 54.9 (d) & \\
\hline $9 \mathrm{a}$ & $3.78(\mathrm{~m})$ & $69.8(t)$ & $C-7^{\prime}, 8^{\prime}$ & $3.80(\mathrm{~m})$ & $71.9(\mathrm{t})$ & $C-7^{\prime}, 8^{\prime}$ \\
\hline $9^{\prime} \mathrm{b}$ & $3.24(\mathrm{dd}, 8.7,8.7)$ & & & $4.20(\mathrm{~m})$ & & \\
\hline $\mathrm{I}^{\prime \prime}$ & $4.53(\mathrm{~d}, 6.7)$ & $65.8(t)$ & $C-4,2^{\prime \prime}, 3^{\prime \prime}$ & $4.56(\mathrm{~d}, 6.1)$ & $65.9(\mathrm{t})$ & $C-4,2^{\prime \prime}, 3^{\prime \prime}$ \\
\hline $2^{\prime \prime}$ & $5.47(t, 6.7)$ & $121.0(\mathrm{~d})$ & $\mathrm{C}-4^{\prime \prime}, \mathrm{C}-5^{\prime \prime}$ & $5.49(\mathrm{t}, 6.1)$ & $120.9(\mathrm{~d})$ & $C-4^{\prime \prime}, 5^{\prime \prime}$ \\
\hline $3^{\prime \prime}$ & & $137.1(\mathrm{~s})$ & & & $140.4(\mathrm{~s})$ & \\
\hline $4^{\prime \prime}$ & $1.72(\mathrm{~s})$ & $17.7(\mathrm{q})$ & $C-2^{\prime \prime}, 3^{\prime \prime}$ & $1.72(\mathrm{~s})$ & $16.2(9)$ & $C-2^{\prime \prime}, 3^{\prime \prime}, 5^{\prime \prime}$ \\
\hline $5^{\prime \prime}$ & $1.75(\mathrm{~s})$ & $25.4(\mathrm{q})$ & $C-2^{\prime \prime}, 3^{\prime \prime}$ & $2.05(\mathrm{~m})$ & $39.8(\mathrm{t})$ & $C-2^{\prime \prime}, 3^{\prime \prime}, 1^{\prime \prime \prime}, 2^{\prime \prime \prime}$ \\
\hline$I^{\prime \prime \prime}$ & & & & $2.10(\mathrm{~m})$ & $26.7(t)$ & $C-2^{\prime \prime}, 3^{m}, 5^{\prime \prime}$ \\
\hline $2^{\prime \prime \prime}$ & & & & $5.11(\mathrm{~m})$ & $124.4(\mathrm{~d})$ & $C-4^{\prime \prime \prime}, 5^{\prime \prime \prime}$ \\
\hline $3^{\prime \prime \prime}$ & & & & & $131.7(\mathrm{~s})$ & \\
\hline $4^{\prime \prime \prime}$ & & & & $1.65(\mathrm{~s})$ & $25.5(\mathrm{q})$ & $\mathrm{C}-2^{\prime \prime \prime}=3^{m \prime}$ \\
\hline $5^{\prime \prime \prime}$ & & & & $1.59(\mathrm{~s})$ & $17.3(\mathrm{q})$ & $C-2^{m \prime}=3^{m}$ \\
\hline $3-\mathrm{OCH}_{3}$ & $3.80(s)$ & $55.6(\mathrm{q})$ & $C-3$ & $3.81(\mathrm{~s})$ & $55.7(9)$ & $C-3$ \\
\hline $3^{\prime}-\mathrm{OCH}_{3}$ & $3.83(s)$ & $55.8(\mathrm{q})$ & $C-3^{\prime}$ & $3.84(\mathrm{~s})$ & $55.8(9)$ & $C-3^{\prime}$ \\
\hline $4^{\prime}-\mathrm{OH}$ & $7.53(\mathrm{~s})$ & & $C-3^{\prime}, 4^{\prime}, 5^{\prime}$ & $7.58(\mathrm{~s})$ & & $C-3^{\prime}, 4^{\prime}, 5^{\prime}$ \\
\hline
\end{tabular}


Co.. Ltd. P. R. China). Column Chromatography (CC): Silica gel (200-300 mesh; Oingdao Haivang Chemical Co., Ltd., P. $\mathrm{R}$. China) and $\mathrm{C}_{18}$ reversed-phase silica gel (YMC CO., LTD. Japan). HPLC: Ultimate 3000 HPLC system(Dionex Co. California. USA): Ultimate 3000 pump: Ultimate 3000 Variable Wavelength; column waters $5 \mathrm{C}_{18}-\mathrm{MS}-\mathrm{II}(10 \times 250 \mathrm{~mm})$. Optical rotation: Perkin-Elmer 341 plolarimeter (Perkin Elmer Inc.. Massachusetts. USA) . ${ }^{1} \mathrm{H}$ - and ${ }^{13} \mathrm{C}-\mathrm{NMR}$ spectral: BrukerAM- 100 instrument (Bruker company. Massachusetts, USA) : ò in ppm rel. to SiMet as internal standard (=0 ppm). $J$ in $\mathrm{Hz}$. EI-MS: Finnigan-MAT-95 mass spectrometer (Finnigan company: UK ) $(70 \mathrm{eV})$; in $m z$ (rel. \%). ESI-MS and HR-ESI-MS data was obtained form Shanghai Institute of Materia Medica. Chinese Academy of Sciences.

Plant material. The barks of Zanthoxylum planispintm were collected from BaDong county. Hubei Province. PR China and identified by Prof. Dinrong Wan. College of Phamacy. South Central University for Nationalities. The voucher specimen (07092701) was deposited in the Herbarium of College of Pharmacy. South Central University for Nationalities

Extraction and isolation. The air-dried bark of Zanthoxy $/ \mathrm{tm}$ planispinum $(17 \mathrm{~kg}$ ) were powdered and then extracted three times with $\mathrm{MeOH}$ at room temperature. and the methanolic extract $(1.6 \mathrm{~kg}$ ) was successively partitioned with petroleum ether. EtOAc and $n-\mathrm{BuOH}$. The EtOAc extract $(47 \mathrm{~g})$ was chromatographed on a silica gel $(800 \mathrm{~g})$ column $(8 \times 50 \mathrm{~cm})$ eluting with a gradient mixture of cyclohexane and acetone (cyclohexane-Me $\mathrm{CO}_{2}$ 95:5, $3 \mathrm{~L}: 9: 1,6 \mathrm{~L}: 8: 2,5 \mathrm{~L}: 7: 3,+\mathrm{L}$ : $1: 1.3 \mathrm{~L} .0: 1.3 \mathrm{~L}$ ) to afford 8 fractions (Frl-Fr8). Fi6 was further chromatographed on a silica gel column $(5 \times 70 \mathrm{~cm})$ with gradient mixture of cyclohexane and ethyl acetate (cyclohexaneEtOAc 9:1. 1.2 L; 8:2. 1.5 L; $7: 3,5 \mathrm{~L}: 1: 1.2 .5 \mathrm{~L} ; 0: 1 .+\mathrm{L})$ to give 6 subfractions (Fr6.1-Fr6.6). Fr6.3 was further purified by $\mathrm{C} 18$ reversed-phase silica gel column $(2 \times 50 \mathrm{~cm})$ with gradient mixture of $\mathrm{H}_{2} \mathrm{O}$ and $\mathrm{MeOH}\left(\mathrm{H}_{2} \mathrm{O}-\mathrm{MeOH} 7: 3,1 \mathrm{~L}\right.$ : 1:1. 2 L, 3:7. $6 \mathrm{~L}: 2: 8,2 \mathrm{~L} .1: 9,1 \mathrm{~L} ; 0: 1.2 \mathrm{~L}$ ) to afford 7 subfractions (Fr6.3.1-Fr6.3.7). Fr6.3.3 was subjected to semiprepared $\mathrm{HPLC}\left(\mathrm{MeOH} / \mathrm{H}_{2} \mathrm{O} 80: 20.3 \mathrm{~mL} / \mathrm{min}: \mathrm{t}_{\mathrm{R}} 3.5 \mathrm{~min}\right.$ ) to afford compound 1 (20 $\mathrm{mg}$ ). Fr6.3.6 were purified by semiprepared $\mathrm{HPLC}\left(\mathrm{MeOH} / \mathrm{H}_{2} \mathrm{O} 70: 30.3 \mathrm{~mL} / \mathrm{min} \mathrm{t}_{\mathrm{R}} 46.7 \mathrm{~min}\right)$ to give compound 2 (40 $\mathrm{mg}$ )
Planispine A (1) colorless oil, $[\alpha]_{D}=-22.3$ (c 0.35 . acetone). EIMS $m z 358$ (100). 205 (25). 163 (35). 151 (85). $137(50)$. 131 (20), 69 (30). HR-ESI-MS $m z 449.1942$ [M+Na] ${ }^{-}$(calculated for $\left.\mathrm{C}_{25} \mathrm{H}_{36} \mathrm{O}_{6} \mathrm{Na} .4+4.1940\right),{ }^{3} \mathrm{H}$ and ${ }^{13} \mathrm{C}-\mathrm{NMR}$ : see Table 1.

Planispine B (2) colorless oil. $[\alpha]_{D}=-5.4$ (c 0.95, acetone). EIMS $m z 388(10), 358$ (100). 327 (15). 205 (15). 163 (35), $151(70) .137(50), 131(15) .12+(10) .69(55)$. HR-ESI-MS $m z 517.2556[\mathrm{M}+\mathrm{Na}]^{-}$(calculated for $\mathrm{C}_{310} \mathrm{H}_{38} \mathrm{O}_{6} \mathrm{Na} .517 .2566$ ) ${ }^{1} \mathrm{H}$ and ${ }^{13} \mathrm{C}-\mathrm{NMR}$ : see Table 1.

Aclonowledgments. We gratefully acknowledge financial support of this work by the National Natural Science Foundation of Cluina (No. 30670215 ).

\section{Refeiences}

1. Fang, Z. X.; Liao, L. C. Hube Enshi Yaovong Zhinuzhi, Hubei Science and Technology Press: WuHan, China, 2006: 577.

2. Tane, P.: Wabo, H. K.; Connolly, J. D. Fitoterapia. 2005, 76. 656.

3. (a) Chen, I. S.: Lin, Y. C.: Tsai, I. L.: Teng. C. M: Ko, F. N.: Ishikawa, T.: Ishii, H. Phytochemismy 1995, 39, 1091 (b) Chang. C. T.; Doong, S. L.; Tsai, I. L.; Chen, I. S. Phutochemisny 1997. 45,1419 .

4. (a) Kalia, N. K.: Singh, B.: Sood, R. P. J. Nat. Prod. 1999, 62, 311. (b) Kashiwada, Y.; Ito, C.: Katagiri, H.: Mase, I.: Komatsu, K.: Namba, T.: Ikeshiro, Y. Phytochemistry 1997, 44, 1125

5. (a) Rahman, M. M.: Islam, M. A.: Khondkar, P:; Gray, A. I. Biochemical Systematics and Ecologv, 2005, 33,91, (b) Chen. I. S.; Chen, T. L.; Chang. Y. L.: Teng. C. M.: Lin, W. Y. J. Kat. Prod. 1999, 62,833.

6. (a) Yang, G. Z.; Li, H. X.: Song, F. I.; Chen, Y. Heh Chim .icta 2007, 90, 1289. (b) Zhong. F. F.: Chen, Y.; Yang. G. Z. Hel Chim Acta 2008, 91, 1695

7. (a) Ahmed, A. A.; Mahmold, A. A.; Ali, T. E.; Tzakou, O.; Couladis, M.: Mabry, T. I.; Gati, T.; Toth, G. Phutochemistn 2002, 59, 851. (b) Lee, H. K. Seo, S. M.; Lee, O. K; Jo, H. J.; Kang, H. Y:- Choi, D. H.; Paik, K. H.; Khan, M. Helv Chim .Acto 2008, 91,2361

8. (a) Zhao, Y. M. Phenylpropanoids; Chemical Industry Press: Beijing China, 2005; p 183, 186. (b) Swain, N. A.: Brown, R. C. D.; Briton, G. J. Org. Chem $\mathbf{2 0 0 4}, 69,123$

9. Hosokawa, A.; Sumino, M.; Nakamura, T.; Yano, S.; Sekine, T.; Ruangrungsi, N.; Watanabe, K.; Ikegami, F. Chent. Pham. Bull. $2004,52,1265$. 